\title{
Two Types of Phase Bunching in the Whistler Mode Wave-Particle Interaction
}

\author{
Hiroshi Matsumoto*, Kozo Hashimoto**, \\ and Iwane KIMURA** \\ *Ionosphere Research Laboratory, \\ Kyoto University, Kyoto, Japan \\ **Department of Electrical Engineering, \\ Kyoto University, Kyoto, Japan
}

(Received April 30, 1974; Revised August 5, 1974)

\begin{abstract}
Importance of the "phase bunching" of resonant electrons in the whistler mode (WM) wave-particle interactions was pointed out by Brice. In this paper, we call attention on the point that there are two different types of phase bunching with two physically distinct phasing mechanisms in the case of the WM wave-particle interaction. One is a longitudinal phase bunching (the Brice type) which is caused by a longitudinal acceleration, while the other is a transverse phase bunching which is caused by a transverse acceleration. The both types are considered separately and examined mathematically and physically. In order to visualize the different characteristics of the two, numerical results on the phasing patterns and the resultant resonant current are shown by computing the nonlinear motion of electrons in a monochromatic WM wave with various initial velocities (36 different initial phase angles $\times 15$ different speed $|\boldsymbol{v}|)$.
\end{abstract}

\section{Introduction}

There have been enthusiastic studies on a problem of the cyclotron interaction between energetic electrons and the whistler mode waves, especially regarding with the generation theory of VLF emissions in the magnetosphere. In a weak nonlinear system, a quasilinear approach holds for waves with a rather wide band spectrum (Drummond and Pines, 1962; Vedenov et al., 1962). As an application of the quasi-linear theory to the VLF emission problem, some studies have been performed (Matsumoto and Kimura, 1971; Ashour-Abdalla, 1972). However, for a problem of the nonlinear interaction between a monochromatic wave and particles, the quasilinear theory becomes invalid so that an alternative self-consistent approach should be desired.

As a first step for the monochromatic wave-particle interaction problem, the exact nonlinear trajectories of electrons in a given monochromatic electromagnetic wave field have been solved (Bell, 1964; Roberts and Buchsbaum, 1964; Laird and Knox, 1965; Lutomirski and Sudan, 1966; Dungey, 1969; 
Ashour-Abdalla, 1970). It was pointed out that a phase trapping is important and essentially different from the case of the electrostatic waves. It should, however, be noticed that the concept of the phase trapping does not completely describe a nonlinear feedback of the resonant current to the wave field. There is another possibility that some resonant electrons gather around a certain phase angle even when the electrons are not phase-trapped in a limited angle.

The purpose of the present paper is to investigate the time behavior of resonant electrons in the whistler mode (WM) monochromatic wave paying a special attention to the phase bunching process as a function of time and its resultant resonant currents. The concept of the phase bunching in the WM wave-particle interaction was first construed by BRICE (1963) and it was adopted in the theories of VLF emissions by many authors (Helliwell, 1967; SUdAN and Oтt, 1971; Dysthe, 1971; NunN, 1971). It is, however, pointed out in the present paper that a phase bunching can be classified into two types by two physically distinct phasing mechanisms as will be mentioned below. This point is relevant and of fundamental importance to theoretical studies of VLF emissions. The distinction of the two mechanisms is little appreciated so that a careful attention should be paid in applying these mechanisms to the VLF emission problem.

Two types of the phase bunching are as follows:

(a) One is the phase bunching due to a longitudinal acceleration parallel to $\boldsymbol{B}_{0}$ by a longitudinal Lorentz force $-e \boldsymbol{v}_{\perp} \times \boldsymbol{B}_{w}$ where $\boldsymbol{B}_{w}$ and $\boldsymbol{v}_{\perp}$ are the wave magnetic field and the perpendicular component of the velocity of an electron to the external magnetic field $\boldsymbol{B}_{0}$. We call this type longitudinal phase bunching hereafter.

(b) The other is the phase bunching due to a transverse acceleration perpendicular to $\boldsymbol{B}_{0}$ by a transverse Lorentz force $-e \boldsymbol{v}_{z} \times \boldsymbol{B}_{w}$ where $\boldsymbol{v}_{z}$ is a component of the velocity parallel to $\boldsymbol{B}_{0}$. We call this type transverse phase bunching shortly.

Though it is well understood that a rigorous solution of the electron motion in the WM wave is described by an elliptic integral, their time-behavior is not given as an explicit function of time. In section 2 , therefore, we begin with a consideration on a behavior of the phase of electrons as an explicit function of time in two extreme cases; (1) a case of very high-pitch-angle electrons for which a longitudinal Lorentz force $-e \boldsymbol{v}_{\perp} \times \boldsymbol{B}_{w}$ becomes a dominant controller, and (2) the other of very low-pitch-angle electrons for which another transverse Lorentz force $-e \boldsymbol{v}_{z} \times \boldsymbol{B}_{w}$ becomes predominant. A comparison between the two different types of phase bunching is given as well as their physical interpretation. In order to show the different characteristics of the two types of phase bunching, numerical computations are carried out in Section 3 on the time-behavior of the phase and the magnitude of the perpendicular velocity of nearly resonant electrons for various initial velocities. The time-behavior of the phases and the resultant 
resonant currents are calculated using the above phase-time solutions. Comparison of the numerical results with the analytical and physical consideration in Sec. 2 is also made.

\section{Two Types of Phase Bunching}

\subsection{Basic equations}

In order to elucidate the characteristics of the phase bunching, we consider a simple situation of the interaction between the energetic electrons and the purely transverse WM monochromatic wave which propagates along an external uniform magnetic field $\boldsymbol{B}_{0}$ in a homogeneous plasma. Neglecting the relativistic effects, the equation of motion of an electron in the wave frame is simply given by

$$
\frac{\mathrm{d} \boldsymbol{v}}{\mathrm{d} t}=-\frac{e}{m} \boldsymbol{v} \times\left(\boldsymbol{B}_{0}+\boldsymbol{B}_{w}\right)
$$

where $m, e$ and $\boldsymbol{v}$ are the electronic mass, charge and velocity. $\boldsymbol{B}_{w}$ is the temporarily constant wave magnetic field vector which rotates spatially along $\boldsymbol{B}_{0}$. Written in terms of the velocity components, we have

$$
\begin{gathered}
\frac{\mathrm{d} v_{\perp}}{\mathrm{d} t}=-\Omega_{1} v_{z} \sin \zeta, \\
\frac{\mathrm{d} v_{z}}{\mathrm{~d} t}=\Omega_{1} v_{\perp} \sin \zeta, \\
\frac{\mathrm{d} \zeta}{\mathrm{d} t}=\Omega_{e}+k v_{z}-\Omega_{1} \frac{v_{z}}{v_{\perp}} \cos \zeta,
\end{gathered}
$$

where $v_{\perp}^{2}=v_{x}^{2}+v_{y}^{2}, \Omega_{1}=e B_{w} / m, \Omega_{e}=e B_{0} / m$ and $\zeta=k z+\theta$ is the angle between $\boldsymbol{v}_{\perp}$ and $\boldsymbol{B}_{w}$ as shown in Fig. 1 in which $\theta=\tan ^{-1}\left(v_{y} / v_{x}\right)$ and $k$ is the wave number (Lutomirski and Sudan, 1966; Sudan and OtT, 1971).

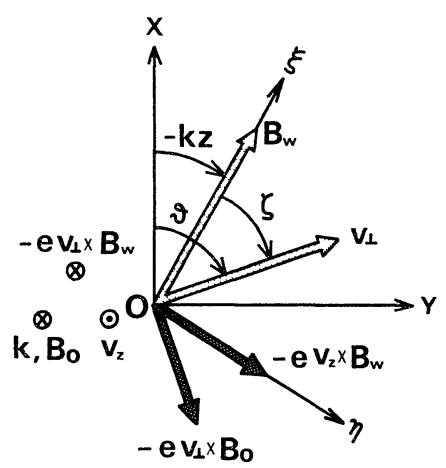

Fig. 1. Wave frame coordinate and three Lorentz forces acting on an electron running antiparallel to $k$. 
In the wave frame, forces acting on the electron are three Lorentz forces, $-e \boldsymbol{v}_{\perp} \times \boldsymbol{B}_{0},-e \boldsymbol{v}_{\perp} \times \boldsymbol{B}_{w}$ and $-e \boldsymbol{v}_{z} \times \boldsymbol{B}_{w}$ as illustrated in Fig. 1. The first Lorentz force $-e \boldsymbol{v}_{\perp} \times \boldsymbol{B}_{0}$ causes the cyclotron motion of the electron and corresponds to the first term in the r.h.s. of (4). The second Lorentz force $-e \boldsymbol{v}_{\perp} \times \boldsymbol{B}_{w}$ accelerates the electron in the longitudinal direction parallel to $\boldsymbol{B}_{0}$ and changes $v_{z}$ through (3) which, in turn, results in the phase change mainly by the second term in the r.h.s. of (4). The third Lorentz force $-e \boldsymbol{v}_{z} \times \boldsymbol{B}_{w}$ is also responsible for changing the phase since it accelerates electrons in the plane perpendicular to $\boldsymbol{B}_{0}$ yielding the change of both the direction and the magnitude of the vector $\boldsymbol{v}_{\perp}$. This effect is expressed by the third term in the r.h.s. of (4).

Since the motion of electron is determined by all three forces in the actual situation, the behavior of the electron becomes complex. In the following, however, we investigate the characteristics of the two types of the phase bunching seperately by considering the two extreme situations in which one of the two Lorentz forces $-e \boldsymbol{v}_{\perp} \times \boldsymbol{B}_{w}$ and $-e \boldsymbol{v}_{z} \times \boldsymbol{B}_{w}$ plays a main role. As a model of the interaction, we take a DAwson's model (1962), i.e. a model in which all of the resonant electrons begin to interact with the given electromagnetic field at $t=0$.

\subsection{Longitudinal phase bunching* (Brice Type)}

In this section, we consider a special case in which the initial value $V_{\perp 0}$ (the subscript 0 indicates initial values hereafter) of the perpendicular speed $v_{\perp}$ of the electron is so large that the change of $v_{\perp}$ can be neglected and also that the transverse force given by the third term in the r.h.s. of (4) can be neglected compared with the longitudinal force. Thus the basic equations, in this case, become

$$
\begin{gathered}
v_{\perp} \simeq V_{\perp 0}=\text { constant } \\
\frac{\mathrm{d} v_{z}}{\mathrm{~d} t}=\Omega_{1} V_{\perp 0} \sin \zeta \\
\frac{\mathrm{d} \zeta}{\mathrm{d} t}=\Omega_{e}+k v_{z}
\end{gathered}
$$

It is well known that the solution of (2)-(4) is expressed by the elliptic function as an implicit function of time $t$. Therefore, topological analyses in phase space have been extensively made so far (e.g., see LUTOMIRSKI and SUdAN, 1966) yielding a concept of "phase trapping".

It is, however, noticed that the phase trapping is quite different from the phase bunching. A concept of phase bunching is made clear only by solving the equation of motion as an explicit function of time. An approximation made above makes it possible to acquire the solution of the equation of motion as an

* This type of the phase bunching is sometimes called 'nonlinear phase bunching', but mathematically a linear treatment is possible as seen in the text in the case that $V_{\perp 0}$ is large enough. 
explicit function of time. Though the behavior of the solution of (6) and (7) is well known, it is also discussed here for completeness.

Eliminating $v_{z}$ from (6) and (7), we get

$$
\frac{1}{2}\left(\frac{\mathrm{d} \zeta}{\mathrm{d} t}\right)^{2}+k^{2} V_{\perp 0} V_{w} \cos \zeta=\mathrm{const} .
$$

where $V_{w}=\Omega_{1} / k$. This is a differential equation with the same form as that for a large amplitude pendulum motion which either oscillates around $\zeta=\pi$ or rotates completely. The solution of $(8)$ is given in the Appendix which shows that resonant electrons with $V_{z 0}=V_{R}\left(=-\Omega_{e} / R\right)$ are all phase-trapped regardless of the initial phase angles, while nearly resonant electrons with $V_{z 0}$ satisfying $0<\left|V_{z 0}-V_{R}\right| \leqq$ $2 \sqrt{V_{\perp 0} V_{w}}$ are either phase-trapped or phase-rotating (i.e., phase-untrapped) depending on their initial phase angles $\zeta_{0}$. It also tells us that quasi-resonant electrons with $V_{z 0}$ satisfying $\left|V_{z 0}-V_{R}\right| \geqq 2 \sqrt{V_{\perp 0} V_{w}}$ are all phase-rotating regardless of their initial phase angles.

The solution of (8) is simplified for the resonant electrons with $V_{z 0}=V_{R}$ and is given by

$$
\cos \frac{\zeta}{2}=\cos \frac{\zeta_{0}}{2} \operatorname{sn}\left[k \sqrt{V_{\perp 0} V_{w}} t+K\left(\cos ^{2} \frac{\zeta_{0}}{2}\right)\right]
$$

where $\operatorname{sn}[$ ] is the Jacobi function and $K$ is the complete elliptic integral of the first kind. Equation (9) shows that the $\zeta-t$ curve of a resonant electron with a certain $\zeta_{0}^{\prime}$ is quite symmetric around $\zeta=\pi$ with that for $\zeta_{0}=2 \pi-\zeta_{0}^{\prime}$. Thus we know that all of the resonant electrons change their phase $\zeta$ towards $\zeta=\pi$. The period $T_{0}$ of the periodic motion (9) is given by

$$
T_{0}=\frac{4 K\left(\cos ^{2} \frac{\zeta_{0}}{2}\right)}{k \sqrt{V_{\perp 0} V_{w}}} .
$$

It should be noticed that the periods of individual resonant electrons with different phase angles $\zeta_{0}$ are different from each other as seen in (10). Therefore the resonant electrons with different initial phase angles can never gather together at the same time. The $K$-value for small $\left|\zeta_{0}-\pi\right|$ can, however, be approximated by $K \sim 2$ so that the bunching time for this longitudinal phase bunching, when most of the resonant electrons gather around $\pi$, is approximately given by

$$
T_{B \| \mid}=\frac{1}{4}\left\langle T_{0}\right\rangle \sim \frac{2}{k \sqrt{V_{\perp 0} V_{w}}}
$$

where $\left\langle>\right.$ denotes the averaged value over $\zeta_{0}$.

Thus we can well conclude that the phases of resonant electrons in the longitudinal phase bunching are not concentrated sharply in a narrow angle around $\zeta=\pi$ but are organized rather dimly around $\zeta=\pi$. A schematic illustration of the time behavior of the phases of resonant and nearly resonant 


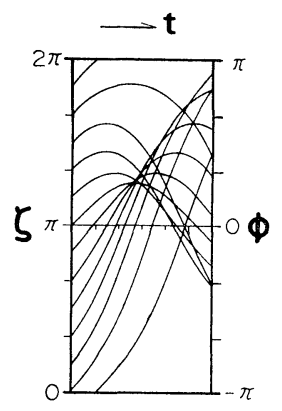

(a)

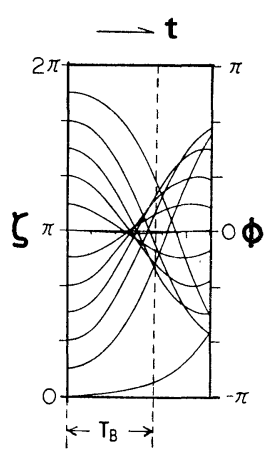

(b)

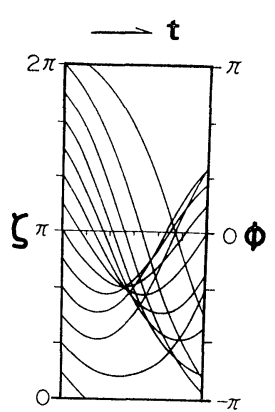

(c)

Fig. 2. Schematic illustration on the phase change due to the longitudinal"phase bunching; (a) $V_{z 0}>V_{R}$, (b) $V_{z 0}=V_{R}$, (c) $V_{z 0}<V_{R}$.

electrons is given in Fig. 2. It is also important to note that the phase re-organization around $\zeta=\pi$ at the second bunching time $t \sim \frac{5}{4} T_{B}$ become more dim than the first phase-bunching.

The physical mechanism of the longitudinal phase bunching was given by BRICE (1963) and is summarized here in what follows. Let us consider the motion of resonant electron in the wave frame which has the initial velocity of sufficiently large $V_{\perp 0}$ and $V_{z 0}=V_{R}$ (See Fig. 1), where $V_{R}$ is the resonance velocity given by $-\Omega_{e} / k$ in the wave frame. Since the electron under consideration has sufficiently large $V_{\perp 0}$, a perturbation on the magnitude $v_{\perp}$ caused by a transverse Lorentz force $-\boldsymbol{e} \boldsymbol{v}_{z} \times \boldsymbol{B}_{w}$ is well negligible. Therefore the longitudinal Lorentz force $-e \boldsymbol{v}_{\perp} \times \boldsymbol{B}_{w}=-e v_{\perp} B_{w} \sin \zeta$ acting upon the resonant electron is mainly controlled only by a phase angle $\zeta$ between $\boldsymbol{v}_{\perp}$ and $\boldsymbol{B}_{w}$ vectors. Namely the resonant electrons with such $\zeta_{0}$ as $0 \leqq \zeta_{0} \leqq \pi$ are decelerated (i.e., $\left|V_{z}\right|$ decreases) while those with $\zeta_{0}$ as $\pi \leqq \zeta_{0} \leqq 2 \pi$ are accelerated (i.e., $\left|V_{z}\right|$ increases). Therefore the time derivative of the phase $\dot{\zeta}$ which initially $\dot{\zeta}=\Omega_{e}+k V_{z 0}=\Omega_{e}+k V_{R}=0$ becomes positive or negative according to $0 \leqq \zeta_{0} \leqq \pi$ or $\pi \leqq \zeta_{0} \leqq 2 \pi$, respectively. Therefore the relative phase angle $\zeta$ changes towards $\zeta=\pi$. When $\zeta$ crosses over $\pi$, the sense of acceleration and deceleration is inverted and therefore the transverse Lorentz force acts as a restoring force of the phase oscillation around $\zeta=\pi$.

We may also describe the above process in the following way. The resonant electrons traversing with $v_{z}=V_{R}$ in the helical magnetic WM wave field first see an almost constant phase because of the matching between the Larmor rotation of $\boldsymbol{v}_{\perp}$ and the Doppler shifted rotation of $\boldsymbol{B}_{w}$. However the electrons are perturbed by the transverse Lorentz force $-e \boldsymbol{v}_{\perp} \times \boldsymbol{B}_{w}$ (or physically identical radiation pressure) yielding a phase change until the electrons suffer from minimum transverse force (or radiation pressure).

\subsection{Transverse phase bunching}

In this section, we consider another extreme case in which $V_{\perp 0}$ is so small, 
that the perturbation of $V_{z}$ by the longitudinal Lorentz force $-e \boldsymbol{v}_{\perp} \times \boldsymbol{B}_{w}$ is negligible, i.e. $v_{z} \simeq V_{z 0}$. Under this first-order approximation, the basic equations become

$$
\begin{gathered}
\frac{\mathrm{d} v_{\perp}}{\mathrm{dt}}=-\Omega_{1} V_{z 0} \sin \zeta \equiv-k V_{z 0} V_{w} \sin \zeta, \\
\frac{\mathrm{d} \zeta}{\mathrm{d} t}=\Omega_{e}+k V_{z 0}-\Omega_{1} \frac{V_{z 0}}{v_{\perp}} \cos \zeta \equiv k\left(V_{z 0}-V_{R}\right)-\frac{k V_{z 0} V_{w}}{v_{\perp}} \cos \zeta,
\end{gathered}
$$

or equivalently

$$
\frac{\mathrm{d}}{\mathrm{d} t}\left(v_{\perp} e^{j \zeta}\right)-j k\left(V_{z 0}-V_{R}\right) v_{\perp} e^{j \zeta}=-j k V_{z 0} V_{w} .
$$

Solutions of (14) are

$$
\begin{aligned}
v_{\xi} & \equiv v_{\perp} \cos \zeta \\
& =\frac{V_{z 0} V_{w}}{V_{z 0}-V_{R}}\left[1-\cos \left\{k\left(V_{z 0}-V_{R}\right) t\right\}\right]+V_{\perp 0} \cos \left\{k\left(V_{z 0}-V_{R}\right) t+\zeta_{0}\right\}, \\
& v_{\eta} \equiv v_{\perp} \sin \zeta \\
& =-\frac{V_{z 0} V_{w}}{V_{z 0}-V_{R}} \sin \left\{k\left(V_{z 0}-V_{R}\right) t\right\}+V_{\perp 0} \sin \left\{k\left(V_{z 0}-V_{R}\right) t+\zeta_{0}\right\}
\end{aligned}
$$

where $v_{\xi}$ and $v_{\eta}$ are the components of $v_{\perp}$ in the directions of $\xi$ axis and $\eta$ axis shown in Fig. 1. From (15) and (16), we get the expressions for the time change of both $v_{\perp}$ and the phase angle $\zeta$ as follows.

and

$$
\zeta=\tan ^{-1}\left[\frac{V_{\perp 0} \sin \left\{k\left(V_{z 0}-V_{R}\right) t+\zeta_{0}\right\}-\frac{V_{z 0} V_{w}}{V_{z 0}-V_{R}} \sin \left\{k\left(V_{z 0}-V_{R}\right) t\right\}}{V_{\perp 0} \cos \left\{k\left(V_{z 0}-V_{R}\right) t+\zeta_{0}\right\}+\frac{V_{z 0} V_{w}}{V_{z 0}-V_{R}}\left[1-\cos \left\{k\left(V_{z 0}-V_{R}\right) t\right\}\right]}\right]
$$

$$
v_{\perp}=\left[\left(k V_{z 0} V_{w} S t\right)^{2}-2 k V_{z 0} V_{w} V_{\perp 0} \sin \left\{\frac{k\left(V_{z 0}-V_{R}\right)}{2} t+\zeta_{0}\right\} S t+V_{\perp 0}^{2}\right]^{1 / 2}
$$

where $S \equiv 2 \sin \left\{k\left(V_{z 0}-V_{R}\right) t / 2\right\} / k\left(V_{z 0}-V_{R}\right) t$ is the sampling function. It should be noticed that the approximation made here is equivalent to the linear calculation discussed by STIX (1962) except for $V_{\perp 0} \neq 0$.

Using these solutions (17) and (18), we can show the second type of the phase bunching which is different from the first longitudinal phase bunching that is discussed in the previous section.

The phase change of the resonant electrons are described by

$$
\zeta=\tan ^{-1}\left\{\tan \zeta_{0}-\frac{k V_{w} V_{R}}{V_{\perp 0} \cos \zeta_{0}} t\right\}
$$

which shows that $\zeta-t$ curves are symmetric around $\zeta=\pi / 2$ and that all of the resonant electrons with different initial phase angle $\zeta_{0}$ are phase-bunched within 
the range of $|\zeta-\pi / 2|<\Delta \zeta$ in a time $T_{B_{\perp}}$ expressed by

$$
T_{B \perp}=\frac{V_{\perp 0}}{k V_{w}\left|V_{R}\right|} \tan \left(\frac{\pi}{2}-\Delta \zeta\right)
$$

Equation (19) means that the phases of all resonant electrons approach to $\zeta=$ $\pi / 2$ closer and closer as time elapses within the first-order approximation that $v_{z}=V_{z 0}=$ constant. A schematic illustration of the transverse phase bunching is given in Fig. 3. Comparing Fig. 3. with Fig. 2, we can well recognize the

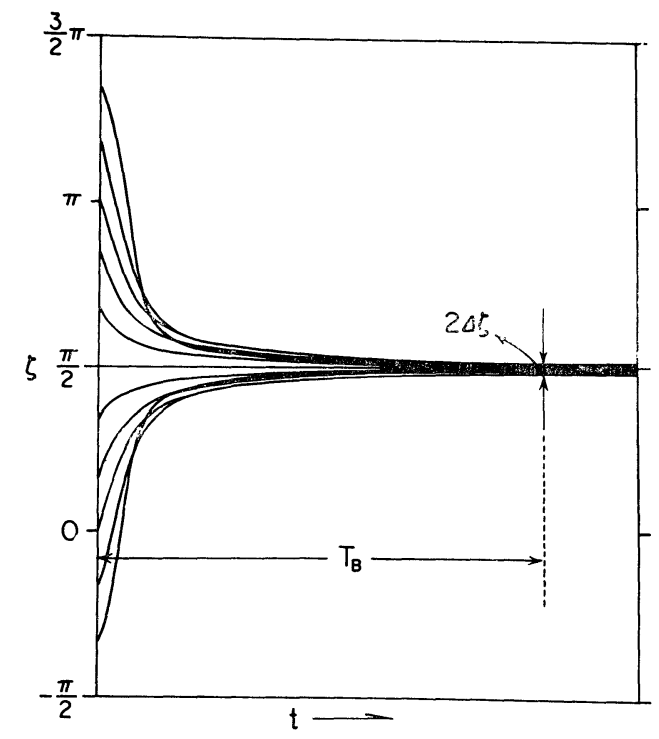

Fig. 3. Schematic illustration of the transverse phase bunching of resonant electrons.

difference of the time behavior of both types of phase bunching. In the case of the transverse phase bunching, different from a dim bunching in the case of the longitudinal phase bunching, the phases are organized tightly around $\zeta=\pi / 2$.

Though the transverse bunching time $T_{B \perp}$ is a function of the bunching tightness $\Delta \zeta$, it is generally shorter than the longitudinal bunching time $T_{B \| \mid}$. As a numerical example, let us consider a WM wave with $f=1 / T=10 \mathrm{kHz}$ and $E=1 \mathrm{mV} / \mathrm{m}$ propagating in a plasma with $f_{p}=180 \mathrm{kHz}, f_{H}=20 \mathrm{kHz}$. Then for resonant electrons $\left(V_{R} \simeq 3.3 \times 10^{7} \mathrm{~m} / \mathrm{s}\right)$ with $V_{\perp 0}=10^{4} \mathrm{~m} / \mathrm{s}$ and $V_{\perp 0}=10^{5}$, $T_{B \perp} \simeq 0.5 T$ and $T_{B \perp} \simeq 5 T$ respectively if we take the bunching tightness $\Delta \zeta$ as $\Delta \zeta=30^{\circ}$. While the resonant electrons with $V_{\perp 0} \simeq 10^{7} \mathrm{~m} / \mathrm{s}$ suffer from the longitudinal phase bunching and $T_{B \|} \simeq 30 T$.

As seen in Fig. 3 the phases $\zeta(t)$ approach monotonously to $\pi / 2$. The corresponding change of $v_{\perp}(t)$ is given by 


$$
v_{\perp}(t)=\left[\left(k V_{w} V_{R} t-V_{\perp 0} \sin \zeta_{0}\right)^{2}+V_{\perp 0}^{2} \cos ^{2} \zeta_{0}\right]^{1 / 2}
$$

which means that $v_{\perp}(t)$ also increases monotonously with time for $t>$ $\left(V_{\perp 0} / k V_{w} V_{R}\right) \sin \zeta_{0}$ within the first-order approximation that $v_{z}=V_{R}=$ const. This is quite different from the time behavior of the longitudinal phase bunching in which both $\zeta(t)$ and $v_{z}(t)$ change periodically interwiring each other. The almost linear acceleration of $v_{\perp}(t)$ is saturated by the neglected longitudinal Lorentz force $-e \boldsymbol{v}_{\perp} \times \boldsymbol{B}_{w}$ becomes innegligible. This point will be discussed in the next section.

The physical mechanism of the transverse phase bunching may be explained as follows. Since the resonant electrons under consideration have small enough $v_{\perp}$, the longitudinal Lorentz force cannot affect the parallel velocity $v_{z}\left(=V_{R}\right)$ and hence the electrons run through the wave field keeping such a matching state that the Larmor rotating frequency $\Omega_{e}$ of $\boldsymbol{v}_{\perp}$-vector equals to the Doppler shifted rotating frequency $-k V_{R}=\Omega_{e}$ of the wave magnetic field $\boldsymbol{B}_{w}$-vector. Therefore the only force that makes the phase $\zeta$ change is the transverse rotating Lorentz force $-\boldsymbol{e} \boldsymbol{v}_{z} \times \boldsymbol{B}_{w}$ which is perpendicular to $\boldsymbol{B}_{w}$ in the transverse plane to $\boldsymbol{B}_{0}$ (See Fig. 1). Therefore all the resonant electrons with $v_{z}=V_{R}$ and small $v_{\perp}$ are made to rotate in the transverse plane to $\boldsymbol{B}_{0}$ towards $\zeta=\pi / 2$. Once the electrons are phase bunched around $\zeta=\pi / 2$, the perpendicular velocity vector $v_{\perp}$ becomes parallel to the transverse Lorentz force and therefore is accelerated until the longitudinal Lorentz force $-e \boldsymbol{v}_{\perp} \times \boldsymbol{B}_{w}$ becomes influential upon the so far constant parallel velocity $v_{z}$. It is noticed here that a part of these resonant electrons with the initial phase angles such as $\pi / 2<\zeta_{0}<(3 / 2) \pi$ never take the exact phase angle $\zeta=\pi / 2$ though it approaches indefinitely closer to $\pi / 2$. This is shown by the following consideration.* From the basic Eqs. (2)-(4), we can get the two constants of motion for electrons.

$$
\begin{gathered}
v^{2}=v_{\perp}^{2}+v_{z}^{2}=\text { const. } \\
\frac{1}{2}\left(v_{z}-V_{R}\right)^{2}+V_{w} v_{\perp} \cos \zeta=\text { const. }
\end{gathered}
$$

Therefore the resonant electrons with initial conditions of $v_{z}=V_{z 0}=V_{R}$ and $\zeta=\zeta_{0}$ should satisfy

$$
V_{w} V_{\perp 0} \cos \zeta_{0}=\frac{1}{2}\left(v_{z}-V_{R}\right)^{2}>0
$$

if $\zeta$ becomes just equal to $\pi / 2$ at later time. This means the phase of the resonant electrons with $\zeta_{0}$ such as $\pi / 2<\zeta_{0}<(3 / 2) \pi$ can never reach just $\pi / 2$.

\subsection{Saturation of $v_{\perp}$-acceleration in the transverse phase bunching}

In the case of the longitudinal phase bunching, $v_{\perp}$ is almost constant. Therefore the magnitude of the perpendicular resonant current $J_{\perp}$ formed by the bunched electrons depends on how densely the phases are bunched around a certain angle. On the other hand, in the case of the transverse phase bunching, the phases of resonant electrons are tightly phase-bunched and all $v_{\perp}$-vectors are

\footnotetext{
* Suggested by a refree.
} 
directed in the same direction. Therefore the resultant resonant current $J_{\perp}$ increases as $v_{\perp}(t)$ increases after the fastened phase bunching is realized. It is then necessary to know the maximum value of $v_{\perp}$ in order to estimate the maximum resonant current intensity.

Before estimating the maximum saturation value of $v_{\perp}(t)$ in the transverse phase bunching, we had better consider first the saturation process and mechanism physically. Let us consider the resonant electrons already phase-bunched around $\zeta=\pi / 2$. In this situation, the electrons are continuously accelerated in the $v_{\perp}$ direction because $\boldsymbol{v}_{\perp} / / \boldsymbol{v}_{z} \times \boldsymbol{B}_{w}$. The causative force of changing the phases $\zeta$ from $\zeta=\pi / 2$ is nearly zero because the relative phase angles $\zeta$ to $B_{w}$ is maintained constant as far as the electrons continue to run with the resonance velocity, while the transverse Lorentz force $-e \boldsymbol{v}_{z} \times \boldsymbol{B}_{w}$ is parallel to $\boldsymbol{v}_{\perp}$ and hence impotent to make the phases rotate. However, when $v_{\perp}(t)$ increases, the phase change toward $\zeta=\pi$ is brought about due to unbalancing between the Larmor rotating motion and the changed Doppler shifted rotation of $\boldsymbol{B}_{w}$. This is physically the same process in the longitudinal phase bunching. It is, however, noticed that in this case all the bunched electrons rotate together and slowly (because $v_{\perp}$ is not yet so large compared with the case of the longitudinal phase bunching) with keeping the bunched state. This is because the phase rotating mechanism depends only upon the present value of $\zeta(\simeq \pi / 2)$ and not upon $\zeta_{0}$ and $V_{\perp 0}$. Since the acceleration of $v_{\perp}$ due to the Lorentz force $-e \boldsymbol{v}_{z} \times \boldsymbol{B}_{w}$ is continued as far as $\zeta<\pi$, the phase rotating mechanism toward $\zeta=\pi$ due to the changing Dopplershift prevails the restoring rotating force $-e \boldsymbol{v}_{z} \times \boldsymbol{B}_{w}$. When $\zeta=\pi$ is reached, the increase of $v_{\perp}$ ceases and $v_{\perp}$ becomes maximum since $v_{\perp}$ is now perpendicular to the so-far accelerating Lorentz force $-e \boldsymbol{v}_{z} \times \boldsymbol{B}_{w}$. At the same time, deviation of $v_{z}$ from $V_{R}$ becomes maximum. After this time, the bunched electrons continue to rotate in the same direction with decreasing $v_{\perp}$ due to the deceleration by the transverse Lorentz force $-e \boldsymbol{v}_{y} \times \boldsymbol{B}_{w}$ (See Fig. 1 again). This is the outline of the existence of the maximum value of $v_{\perp}(t)$ in the transverse phase bunching.

The maximum value of $v_{\perp}-v_{\perp \max }-$ and the maximum deviation of the parallel velocity from $V_{R}$ of the resonant electrons can be derived as follows. As considered physically, the main controller of the $v_{\perp}$ acceleration or deceleration is not the magnitude of the transverse Lorentz force $-e \boldsymbol{v}_{z} \times \boldsymbol{B}_{w}$ but its relative phase angle to the perpendicular velocity $v_{\perp}$ of the resonant electrons. Therefore approximating the magnitude of the transverse Lorentz force by $-e V_{z 0} B_{w}$ in the r.h.s. of (2) and the third term of the r.h.s. of (4), we get the following set of equations.

$$
\begin{gathered}
\frac{\mathrm{d} v_{\perp}}{\mathrm{d} t}=-k V_{w} V_{z 0} \sin \zeta \\
\frac{\mathrm{d} v_{z 1}}{\mathrm{~d} t}=k V_{w} v_{\perp} \sin \zeta
\end{gathered}
$$




$$
\frac{\mathrm{d} \zeta}{\mathrm{d} t}=k\left(V_{z 0}-V_{R}\right)+k v_{z 1}-\frac{k V_{z 0} V_{w}}{v_{\perp}} \cos \zeta
$$

where $v_{z 1}$ is a perturbation expressing the deviation of the parallel velocity from $V_{z 0}$. From these equations, we get

$$
\begin{gathered}
v_{z 1}=-\frac{v_{\perp}^{2}-V_{\perp 0}^{2}}{2 V_{z 0}}, \\
v_{\perp} \cos \zeta-V_{\perp 0} \cos \zeta_{0}=\frac{v_{\perp}^{2}-V_{\perp 0}^{2}}{4 V_{z 0} V_{w}}\left\{2\left(V_{z 0}-V_{R}\right)+v_{z 1}\right\} .
\end{gathered}
$$

When $v_{\perp}$ increases so that $v_{\perp} \gg V_{\perp 0},(29)$ is well approximated for the resonant electrons as

$$
v_{\perp} \cos \zeta-V_{\perp 0} \cos \zeta_{0}=-\frac{v_{\perp}^{4}}{8 V_{w} V_{R}^{2}} .
$$

Therefore, when the bunched phase angle $\zeta$ suffers a change far from $\pi / 2$ due to the change of $v_{z}$ so as to satisfy $|\cos \zeta| \gg V_{\perp 0} / v_{\perp}$, the second term in (30) can be neglected to yield

$$
v_{\perp}=-2\left(V_{w} V_{R}^{2} \cos \zeta\right)^{1 / 3}
$$

which shows that the bunched phase angle $\zeta$ changes slowly so as to satisfy $\cos \zeta<0$, i.e., in the direction of $\zeta=\pi$. Thus $v_{\perp}$ does not increase indefinitely with time but reaches a maximum value $v_{\perp \max }$ given by

$$
v_{\perp \max }=2\left(V_{w} V_{R}^{2}\right)^{1 / 3}
$$

when $\zeta=\pi$. The perturbation $\left|v_{z 1}\right|$, is also maximized at the same time as

$$
\begin{aligned}
\left|v_{z 1 \max }\right| & =2\left(V_{w}^{2} V_{R}^{4} V_{z 0}^{-3}\right)^{1 / 3} \\
& \simeq 2\left(V_{w}^{2} V_{R}\right)^{1 / 3} .
\end{aligned}
$$

It should be noticed that a time-behavior of all the resonant electrons is described universally by (31) and (32) regardless not only of their initial phase angles $\zeta_{0}$ but also of their initial perpendicular velocity $V_{\perp 0}$.

These results may be confirmed by looking a numerical example. A polar plot in the $\left(v_{\perp}, \zeta\right)$ polar graph of the numerical solution of the exact nonlinear equation of motion (2)-(4) is given in Fig. 4. In the figure, six different trajectories of resonant electrons with six different $V_{\perp 0}$ values and $\zeta_{0}=\pi$ are illustrated. The end points of the trajectories are the $\left(v_{\perp}, \zeta\right)$ values at the same time. As seen in the figure, the phases are bunched in the same direction regardless of $V_{\perp 0}$. The estimated maximum value $v_{\perp \max }$ given by (32) is $v_{\perp \max } \simeq 2.92 \times 10^{6}$ $(\mathrm{m} / \mathrm{s})$ for the parameters used in the numerical computation. It agrees well with the $v_{\perp \max }$ values especially with the value for $V_{\perp 0}=10^{4} \mathrm{~m} / \mathrm{s}$ in Fig. 4 .

As for the quasiresonant electrons which satisfies $\left|V_{z 0}-V_{R}\right| \gg v_{z 1 \max }$ or equivalently 


$$
\left|\frac{V_{z 0}-V_{R}}{V_{R}}\right| \gg 2\left(V_{w} / V_{R}\right)^{1 / 3},
$$

the perturbation $v_{z 1}$ can be neglected so that the first-order approximation made in the previous section is effective. The change of the phase of the quasiresonant electrons is almost the same as the resonant electrons for small $t$, but is described approximately by

$$
\zeta=\frac{k\left(V_{z 0}-V_{R}\right)}{2} t+\frac{\pi}{2}
$$

after a time when the first terms in both the numerator and the denominator in

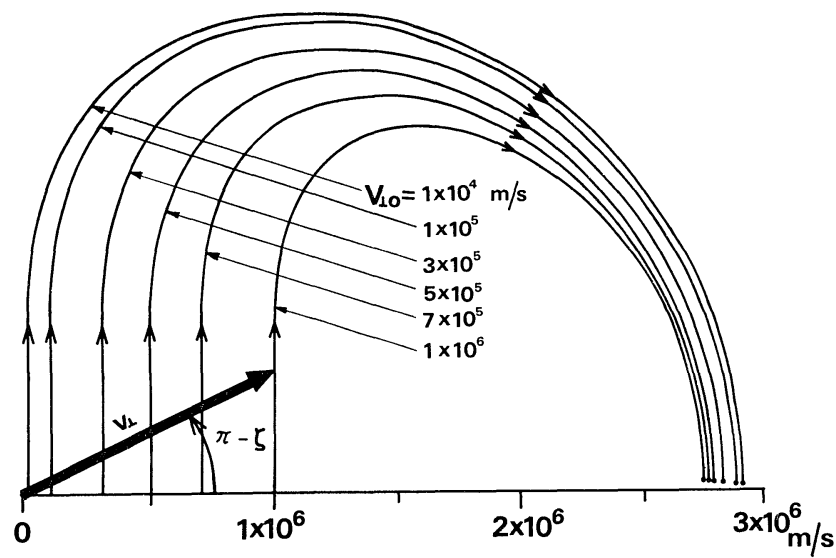

Fig. 4. Polar representation of the locus of the perpendicular velocity $\left(V_{\perp}, \zeta\right)$ in case of the transverse phase bunching. Computations were done under the following parameters; $V_{R}=-3.328 \times 10^{7} \mathrm{~m} / \mathrm{s}, \quad V_{w}=2.798 \times 10^{3} \mathrm{~m} / \mathrm{s}, f_{p}=$ $180 \mathrm{kHz}, f_{H}=20 \mathrm{kHz}, f=10 \mathrm{kHz}$.

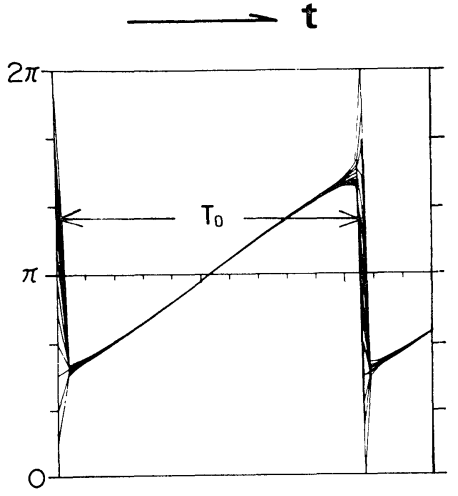

(a) $V_{Z O}>V_{R}$

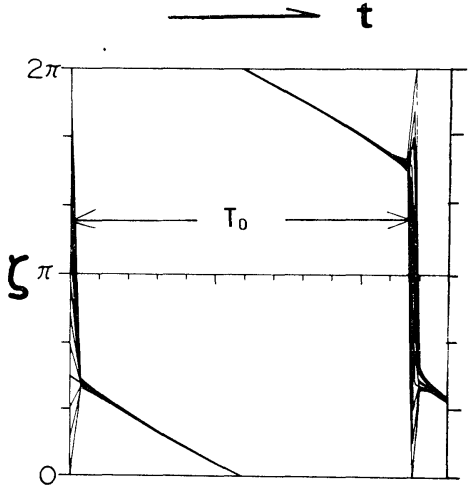

(b) $\mathrm{V}_{\mathrm{zO}}<\mathrm{V}_{\mathrm{R}}$

Fig. 5. Schematic illustration of the transverse phase bunching of quasiresonant electrons. 
(17) can be neglected. Noting that $\zeta\left(t, \zeta_{0}\right)=-\zeta\left\{2 \pi / k\left(V_{z 0}-V_{R}\right)-t,-\zeta_{0}\right\}$ and $v_{\perp}\left(t, \zeta_{0}\right)=v_{\perp}\left\{2 \pi / k\left(V_{z 0}-V_{R}\right)-t, \zeta_{0}\right\}$ from (17) and (18), we know the time behavior of $v_{\perp}$ and $\zeta$ of the quasiresonant electrons and their period $T_{0}$ which is given by

$$
T_{0}=\frac{2 \pi}{k\left(V_{z 0}-V_{R}\right)}
$$

We can thus show a schematic illustration of the phase change for the quasiresonant electrons as shown in Fig. 5. The maximum value $v_{\perp \max }$ for the quasiresonant electrons which satisfy (34) is given by

$$
v_{\perp \max }=\frac{V_{w} V_{z 0}}{V_{z 0}-V_{R}} .
$$

\section{Numerical Computation}

A numerical computation of the nonlinear basic Eqs. (2) to (4) is performed for various $V_{\perp 0}, V_{z 0}$, and $\zeta_{0}$ in order to visualize a time behavior of the phase $\zeta(t)$ and the perpendicular velocity $v_{\perp}(t)$. An observation is also made on the formation of the resonant current for each initial speed $\left(V_{\perp 0}, V_{z 0}\right)$. Selecting 36 initial phases $\zeta_{0}$ 's uniformly between $\zeta_{0}=0$ to $2 \pi$ for each fixed parameter $\left(V_{\perp 0}\right.$, $\left.V_{z 0}\right)$, a calculation of

and

$$
I_{\xi}\left(V_{\perp 0}, V_{z 0}, t\right)=-e \sum_{i=1}^{36} v_{\perp i} \cos \zeta_{i}
$$

$$
I_{\eta}\left(V_{\perp 0}, V_{z 0}, t\right)=-e \sum_{i=1}^{36} v_{\perp i} \sin \zeta_{i}
$$

is made for $0 \leqq t \leqq 200 T$ where the suffix $i$ refers to different electrons with different initial phase $\zeta_{0}$. For the velocity parameters, three kinds of $V_{z 0}$ were selected as $V_{z 0}<V_{R}, V_{z 0}=V_{R}$ and $V_{z 0}>V_{R}$ and $V_{\perp 0}$ was chosen as five values of $V_{\perp 0}=10^{4}, 10^{5}, 10^{6}, 5 \times 10^{6}$ and $10^{7} \mathrm{~m} / \mathrm{s}$. The results of the computation are shown in Fig. 6 on the phase change and in Fig. 7 on the change of $I_{\xi}$ and $I_{\eta}$ for 15 combined parameters $\left(V_{\perp 0}, V_{z 0}\right)$. Plasma and wave parameters used in the computation were typical values of the whistler mode waves propagating in the magnetosphere. These values are given in the figure captions.

It should be noticed here that the expression for the current calculation is possible only when the electrons are not scattered in space. In cases that the pitch angle of electrons are so large that the longitudinal phase bunching becomes dominant, electrons with different initial phases are scattered in space as time elapses. Therefore, the efficiency of current formation in a given position due to the longitudinal phase bunching becomes worse due to this scattering effect than estimated by (38) and (39). In the case of the transverse phase bunching the spatial scattering is not brought about.

As seen in Fig. 6, the transverse phase bunching is seen for small $V_{\perp 0}$, and 

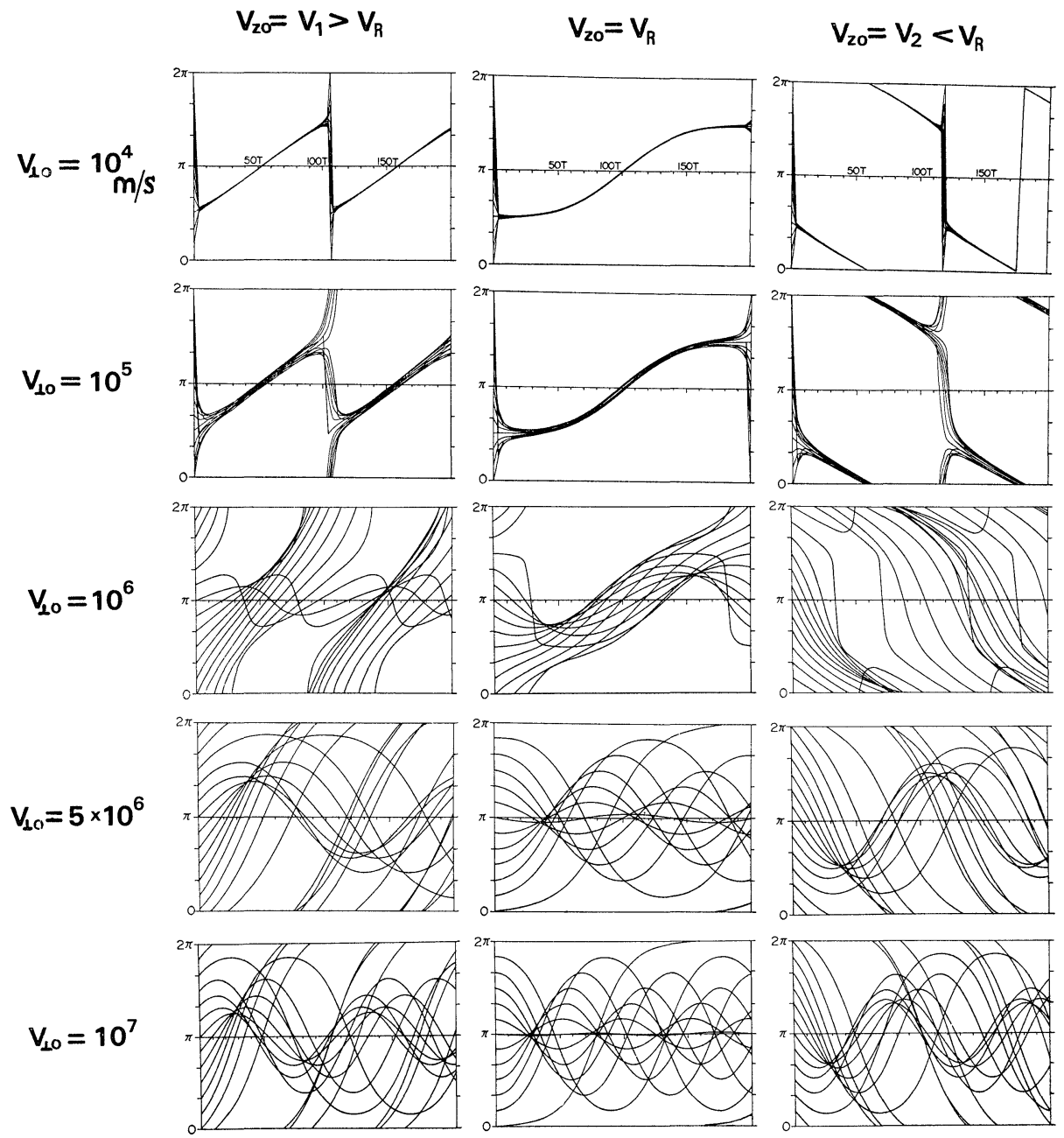

Fig. 6. Phase change with time for various initial speeds $\left(V_{\perp 0}, V_{z 0}\right)$. Computations were done for $0 \leqq t \leqq 200 T$ under the following parameters; $f_{p}=180 \mathrm{kHz}, f_{H}=20 \mathrm{kHz}$, $f \equiv 1 / T=10 \mathrm{kHz}, V_{R}=-3.328 \times 10^{7} \mathrm{~m} / \mathrm{s}, V_{1}=-3.314 \times 10^{7} \mathrm{~m} / \mathrm{s}, V_{2}=-3.344 \times 10^{7} \mathrm{~m} / \mathrm{s}$. Plottings are made for 12 different initial angles, one in every $\pi / 6$ in each figure.

longitudinal phase bunching is, on the contrary, seen for large $V_{\perp 0}$. A transient change from the transverse phase bunching to the longitudinal phase bunching is also observed. Specific characteristics of both types of phase bunching seen in Fig. 6 coincide well with those discussed by an analytical speculation in the previous section.

Figure 7 indicates a corresponding resonant current formed by 36 electrons due to the phase bunching. All the ordinate units are the same in the figure. Comparing the cases of $V_{\perp 0}=10^{4}(\mathrm{~m} / \mathrm{s})$ and of $V_{\perp 0}=10^{7}(\mathrm{~m} / \mathrm{s})$, we can know that the resonant current by the longitudinal phase bunching is only 2 to 4 times 

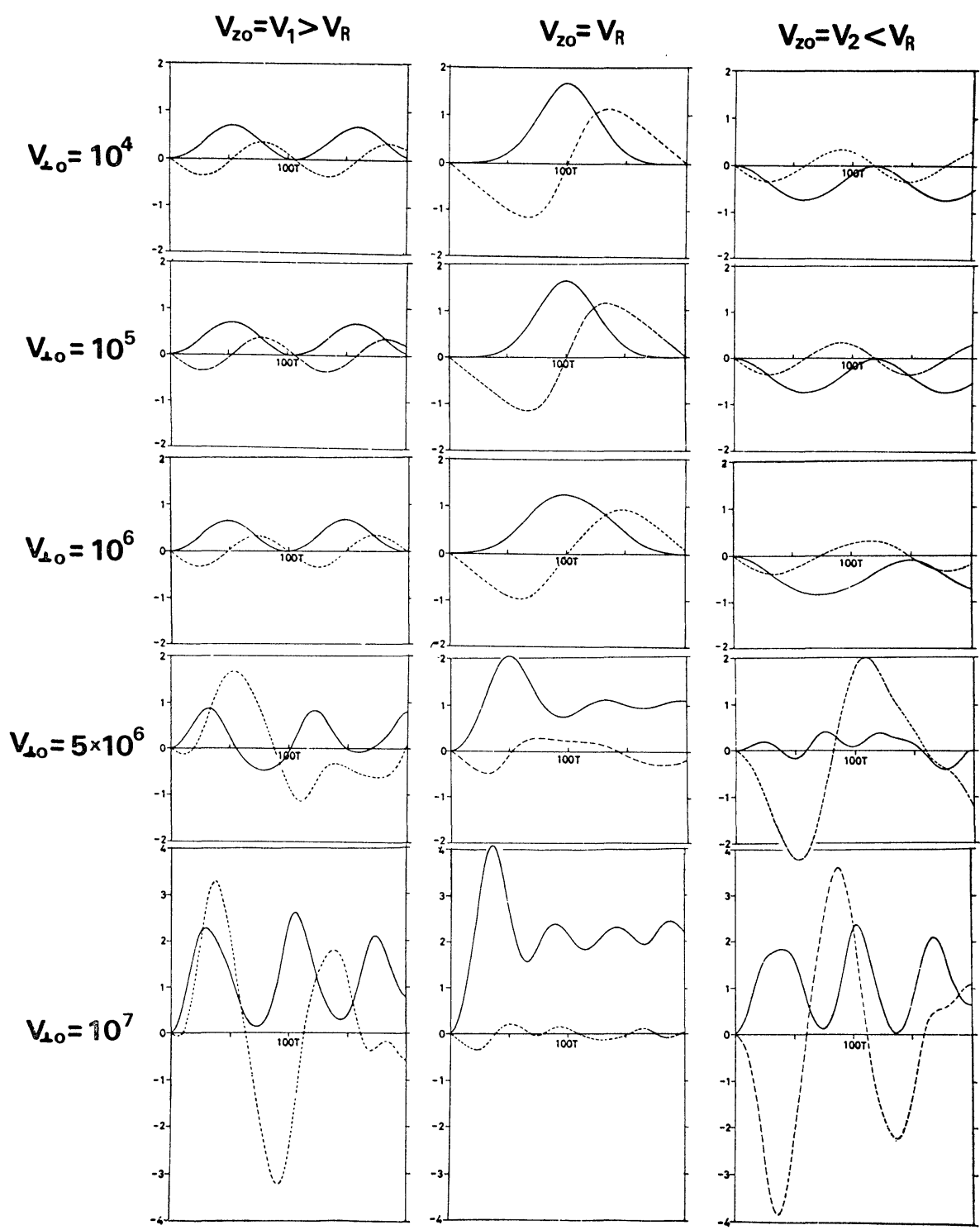

Fig. 7. Corresponding resonant current due to the phase bunching in Fig. 6. Solid and dashed lines indicate $I_{\xi}$ and $I_{\eta}$, respectively.

that by the transverse phase bunching in spite of the 1000 times initial values of $V_{\perp 0}$. It is interesting to note that the time-behavior of the resonant currents $I_{\xi}$ and $I_{\eta}$ does not depend upon $V_{\perp 0}$ for $V_{\perp 0}=10^{4}$ to $10^{6}(\mathrm{~m} / \mathrm{s})$ and shows a resonant character that is $I_{i}(i=\xi, \eta)$ is maximum at $V_{z 0}=V_{R}$ and decreases as $V_{z 0}$ departs from $V_{R}$ with increasing its period. As understood by the previous analyses, $I_{\eta}$ precedes $I_{\xi}$ for small $t$ in the case of small $V_{\perp 0}$. On the contrary, in the case of 
$V_{\perp 0}=5 \times 10^{6}$ and $10^{7}(\mathrm{~m} / \mathrm{s})$, the perpendicular current $I_{\perp}=\left(I_{\xi}^{2}+I_{\eta}^{2}\right)^{1 / 2}$ does not show a peaky resonant character since $I_{\eta}$ becomes minimum at $V_{z 0}=V_{R}$ though $I_{\xi}$ shows a broad resonant character. It should be noticed that a current due to the longitudinal phase bunching shows a nonperiodic behavior as seen in the case of $V_{\perp 0}=5 \times 10^{6}$ and $10^{7}(\mathrm{~m} / \mathrm{s})$ in Fig. 7. This is due to the fact that the period of each electron differs from each other since the period is dependent on the initial phases $\zeta_{0}$.

\section{Discussion}

The nonlinear motion of electrons in a large amplitude electromagnetic wave has been studied so far mainly in the phase domain yielding a concept of the phase trapping similar to the potential trapping in the electrostatic case. The concept of the phase bunching is similar to that of the phase trapping but is different in the sense that the former is defined by the time behavior of phases while the latter is defined only in the topological pattern in phase space.

An analysis of the time behavior of resonant electrons is therefore important. In this paper, we demonstrated that the phase bunching is brought about even when the electrons are not phase-trapped in phase space. In other words, if we pay our attention only to the phase-space behavior of resonant electrons, we lose sight of the fact of the phase bunching under certain plasma parameters. By an analysis of the time behavior of phases of resonant electrons, we have clarified that there exist two types of phase bunching. One is the longitudinal phase bunching (Brice type), the other is the transverse phase bunching, the latter of which could not been found only by a topological analysis in phase space. Electrons with large pitch angles experience mainly the former while electrons with small pitch angle suffer principally the latter.

In a case of a plasma composed of relatively high pitch angle electrons, the longitudinal phase bunching prevails. In this case, the electrons would not be accelerated or decelerated continuously and hence only a small periodic perturbation of the amplitude of the original whistler mode wave is expected. This is just the case of the nonlinear large amplitude oscillation of a whistler mode wave with almost constant amplitude (Palmadesso and Schmidt, 1971). It should, however, be noticed that the present treatment is based on a spatially homogeneous model and therefore a spatial feedback is not taken into account. Recently, HeLliwell and Crystal (1973) presented a self-consistent WM waveparticle interaction theory which considers a feedback of the resonant current due to the longitudinal phase bunching to the WM wave field in both time and space. Their results show an exponential growth of the field which could never be predicted by the above-mentioned homogeneous treatments.

On the contrary, for a plasma in which low pitch electrons dominate, the transverse phase bunching becomes conspicuous. In this case, the acceleration 
of $v_{\perp}$ and the tight phase bunching will form a rapidly growing resonant current yielding a considerable damping of the original whistler mode wave. Therefore a quite different nonlinear behavior would be expected which will be given in another paper. When we limit our attention of the application to the magnetospheric whistler phenomena, it is considered that there exist little resonant electrons with small pitch angles in the magnetospheric mirror field. However, there are two possible cases in which low pitch resonant electrons are produced in the magnetosphere. One is the case of the artificial beam injection from a rocket-borne electron gun into the magnetosphere. The injected electrons could not have enough large pitch angles at the equator and hence suffer from the transverse phase bunching rather than the longitudinal phase bunching*. The another case is that such a low pitch resonant electrons are produced by an acceleration of thermal electrons by a transient field-aligned electric field. In both cases, these low pitch electrons will be soon precipitated into the ionosphere unless they encounter a large amplitude whistler mode wave. However, if they encounter the large amplitude whistler mode wave, as analyzed in the present paper, they will be accelerated in the $v_{\perp}$ direction with being phase-bunched tightly. Thus there may be a case that the transverse phase bunching pointed out in the present paper is realized.

In any case, which phasing mechanism is relevant to the actual WM waveparticle interaction should be carefully considered by investigating the competing process between the two different phasing mechanisms and relating population of resonant electrons with different pitches in different regions of the magnetosphere for various wave amplitude under interest.

We wish to express our thanks to Professor K. Maeda for his valuable discussion and advice. We would like to record our thanks to Professors T. Obayashi, K. Nishikawa, H. Oya and T. Sato for helpful discussions. Thanks are also-due to refrees for enlightening comments and suggestions, and to Messers. K. Mamiya, S. Yokoi and M. Kawai for helping numerical computations in part. All computations were done at the Data Center, Kyoto University.

Appendix. Solution of Eq. (8).

Transforming $\zeta$ into $\phi$ as $\phi=\zeta+\pi$, the solution of (8) is given by

$$
\sin \frac{\phi}{2}=\sin \frac{\phi_{m}}{2} \operatorname{sn}\left[\begin{array}{l}
\left.\operatorname{sgn}\left(V_{z 0}-V_{R}\right) k \sqrt{V_{\perp 0} V_{w}} t+S n^{-1}\left(\frac{\sin \frac{\phi_{0}}{2}}{\sin \frac{\phi_{m}}{2}}\right)\right] \\
\text { for } 0 \leqq\left|\phi_{0}\right| \leqq \phi_{0 L}
\end{array}\right.
$$

or

* It is pointed by a refree that another example would be during a natural injection event, when electrons of all pitch angles are suddenly introduced. 


$$
\begin{gathered}
\sin \frac{\phi}{2}=\operatorname{sn}\left[\operatorname{sgn}\left(V_{z 0}-V_{R}\right) k \sqrt{V_{\perp 0} V_{w}} C t+S n^{-1}\left(\sin \frac{\phi_{0}}{2}\right)\right] \\
\text { for } \phi_{0 L} \leqq\left|\phi_{0}\right| \leqq \pi
\end{gathered}
$$

where $\operatorname{sgn}(0) \equiv 1$ and

$$
\begin{gathered}
\phi_{0 L}=\left\{\begin{array}{cc}
\cos ^{-1} \frac{\left(V_{z 0}-V_{R}\right)^{2}-2 V_{\perp 0} V_{w}}{2 V_{\perp 0} V_{w}} & \text { when }\left(V_{z 0}-V_{R}\right)^{2} \leqq 4 V_{\perp 0} V_{w} \\
0 & \text { when }\left(V_{z 0}-V_{R}\right)^{2} \geqq 4 V_{\perp 0} V_{w}
\end{array}\right. \\
C=\sqrt{\sin ^{2} \frac{\phi_{0}}{2}+\frac{\left(V_{z 0}-V_{R}\right)^{2}}{4 V_{\perp 0} V_{w}}}
\end{gathered}
$$

The solutions (A.1) and (A.2) correspond to the case of the phase trapping and that of the phase rotation respectively. $\phi_{m}$ in (A.1) means the maximum amplitude of the phase oscillation of the phase-trapped electrons and is determined by

$$
\dot{\phi}_{m}=2 \sin ^{-1} C
$$

$S n^{-1}(u)$ is the principal value of the inverse Jacobi function, i.e., $-K \leqq S n^{-1}(u) \leqq$ $K$ where $K$ is the complete elliptic integral of the first kind. The modulus $k_{m}$ of the sn-function in (A.1) and (A.2) determines the period of the phase oscillation by $k_{m}=C$ for the phase-trapped electrons in case of (A.1) and that of the phase rotation by $k_{m}=1 / C$ in case of (A.2).

The resonant electrons are all phase-trapped regardless of the initial phase angle $\phi_{0}$ since $\phi_{m}=\left|\phi_{0}\right|$ from (A.4) and (A.5), and $\phi_{0 L}=\pi$ from (A.3). Using the relations $S n^{-1}( \pm 1)= \pm K$ and $\operatorname{sn}(u+2 K)=-\operatorname{sn}(u)$, the solution for the resonant electrons is simply expressed by

$$
\sin \frac{\phi}{2}=\sin \frac{\phi_{0}}{2} \operatorname{sn}\left[k \sqrt{V_{0 \perp} V_{w}} t+K\right]
$$

\section{REFERENCES}

Ashour-Abdalla, M., Nonlinear particle trajectories in a whistler mode wave packet, Planet. Space Sci., 18, 1799-1812, 1970.

Ashour-Abdalla, M., Amplification of whistler waves in the magnetosphere, Planet. Space Sci., 20, 639-662, 1972.

BeLl, T., Wave-particle gyroresonance interaction in the earth's outer ionosphere, Tech. Rept. No. 3412-5, Stanford University, Stanford Calif., 1964.

Brice, N.M., An explanation of triggered VLF emissions, J. Geophys. Res., 68, 4626-4628, 1963.

Dawson, J., One-dimensional plasma model, Phys. Fluids, 5, 445-459, 1962.

Drummond, W.E. and D. Pines, Nonlinear stability of plasma oscillations, Nucl. Fusion, 2, 1049-1057, 1962.

Dungey, J.W., The motion of a charged particle in the field of a whistler mode wave packet, in Plasma Waves in Space and in the Laboratory, edited by Thomas and Landmark, vol. I, pp. 407-415, Edinburgh University Press, 1969. 
Dysthe, K.B., Some studies of triggered whistler emissions, J. Geophys. Res., 76, 6915-6931, 1971.

Gendrin, R., Phase bunching and other nonlinear processes occurring in gyroresonant waveparticle interactions, Astrophys. Space Sci., 28, 245-266, 1974.

Helliwell, R.A., A theory of discrete VLF emissions from the magnetosphere, J. Geophys. Res., 72, 4773-4790, 1967.

Helliwell, R.A. and T.L. Crystal, A feedback model of cyclotron interaction between whistler mode waves and energetic electrons in the magnetosphere, J. Geophys. Res., 78, 7357-7371, 1973.

LAIRD, M.J. and F.B. KNox, Exact solution for charged particle trajectories in a electromagnetic field, Phys. Fluids, 8, 755-756, 1965.

Lutomirski, R.F. and R.N. Sudan, Exact nonlinear electromagnetic whistler modes, Phys. Rev., 147, 156-165, 1966.

MAtsumoto, H. and I. Kimura, Linear and nonlinear cyclotron instability and VLF emissions in the magnetosphere, Planet. Space Sci., 19, 567-608, 1971.

NunN, D., A theory of VLF emissions, Planet. Space Sci., 19, 1141-1167, 1971.

Palmadesso, P. and G. Schmidt, Collisionless damping of a large amplitude whistler wave, Phys. Fluids, 14, 1411-1418, 1971.

Roberts, C.S. and S.J. Buchsbaum, Motion of charged particle in a constant magnetic field and a transverse electromagnetic wave propagating along the field, Phys. Rev., 135, A381A389, 1964.

Stix, T.H., The Theory of Plasma Waves, MacGraw-Hill, New York, 1962.

Sudan, R.N. and E. Otт, Theory of triggered VLF emissions, J. Geophys. Res., 76, 4463-4476, 1971.

Vedenov, A.A., E.P. Velikov and R.Z. SAgdeev, Quasilinear theory of plasma oscillations, Nucl. Fusion Suppl., 2, 465-475, 1962. 